\title{
Therapieoptionen bei Multipler Sklerose
}

\author{
Isabel Ringel, Uwe Klaus Zettl \\ Klinik und Poliklinik für Neurologie der Universität Rostock
}

psychoneuro 2003; 29 (7+8): 336-343

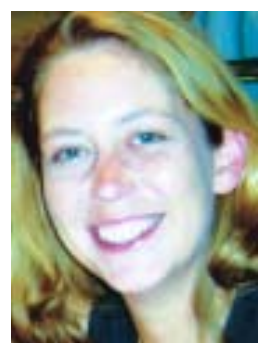

Isabel Ringel
Bei kaum einer anderen neurologischen Erkrankung haben sich die therapeutischen Möglichkeiten in den letzten fünf Jahren so gravierend verändert wie bei der Multiplen Sklerose (MS). Es stehen mit den Beta-Interferonpräparaten und mit Glatirameracetat Basistherapeutika für verschiedene Verlaufsformen der MS zur Verfügung. Selbst für therapieresistente Fälle oder für dramatische Verlaufsformen gibt es evidenz-basierte Möglichkeiten einer Eskalationstherapie. Dabei kommen Immunsuppressiva wie Mitoxantron oder Cyclophosphamid zum Einsatz, aber auch eine Plasmapherese kann in Einzelfällen eine sinnvolle Therapieoption darstellen. Neuere Therapieansätze versuchen jedoch nicht nur das Immunsystem zu supprimieren oder modulieren. Neuroprotektion und Neurodegeneration bei der MS sind Therapieziele zukünftiger Medikamente. tralen Nervensystems, die zumindest bei Erkrankungsbeginn bei $80 \%$ der Patienten durch Schübe gekennzeichnet ist.

Bei Schüben kommt es subakut zu sich entwickelnden neurologischen Ausfällen, die definitionsgemäß mindestens 24 Stunden bestehen und sich von selbst komplett oder inkomplett zurück bilden können. Davon abgegrenzt werden müssen neurologische Ausfälle, die infekt- oder fieberassoziiert sind oder auf anders verursachte Erhöhung der Körpertemperatur (UhthoffPhänomen) zurückzuführen sind (10). Das Therapieziel bei der Schubbehandlung besteht in der Abkürzung des akuten Schubes, der Minderung des Schweregrades der schubbedingten neurologischen Ausfälle sowie der Verzögerung der Behinderungsprogression.

\section{Glukokortikoide}

Die einzige derzeit etablierte Schubtherapie besteht in der hochdosierten Glukokortikosteroidgabe. Da bisher noch keine vergleichende Studie hinsichtlich der Wirksamkeit unterschiedlicher Steroide beim MSSchub erfolgt ist, können für die Auswahl eines bestimmten Glukokortikosteroids nur allgemeine, 
pharmakologische Aspekte herangezogen werden. So besteht bei Methylprednisolon und Dexamethason eine geringere mineralokortikoide Wirkung bei gleichzeitig höherer Kortikoid-Rezeptoraffinität verglichen mit Prednisolon, darüber hinaus eine höhere Liquorgängigkeit und eine lineare Dosis-WirkungKinetik (11). Die Wirkung der Glukokortikosteroide beruht auf genomischen und nicht-genomischen Effekten, wobei nur die nicht-genomischen Wirkungen, die erst ab einer Tagesdosis von $500 \mathrm{mg}$ zu erwarten sind, einen sofortigen Effekt zeigen. Die Wirkung beruht auf antiödematösen, antiinflamatorischen, immunsuppressiven Mechanismen sowie auf der Induktion insbesondere der T-Zellapoptose und auf einer Membranstabilisierung (6).

In der Praxis hat sich die intravenöse Gabe von 1g Methylprednisolon täglich an 3-5 aufeinander folgenden Tagen bewährt (13). Der Nutzen einer oralen Ausschleichphase ist nur für die Behandlung der Optikusneuritis belegt (3) und sollte in Abhängigkeit vom Ansprechen auf die i.v.-Gabe und der individuellen Nebenwirkungsrate entschieden werden. Manche Neurologen tendieren dazu, bei sehr leichten Schüben, gekennzeichnet beispielsweise durch eine rein sensible Symptomatik, auf eine Glukokortikosteroidgabe zu verzichten. $\mathrm{Zu}$ bedenken ist allerdings bei dieser Entscheidung, dass es sich bei dem klinisch sichtbaren Symptom nur um die „Spitze des Eisbergs" der momentan ablaufenden Entzündung handelt und dass bei einer sich entwickelnden Schubsymptomatik nicht absehbar ist, welche persistierenden hirnmorphologische Schäden gesetzt werden.

Empfehlenswert ist, die hochdosierte Steroidgabe stationär durchzuführen, um auf die eventuellen Nebenwirkungen wie eine diabetische Stoffwechsellage, Blutdruckkrisen, Infektprovokation, gastrale Reizung, Thromboseentstehung oder psychotische Entgleisungen entsprechend reagieren zu können. Eine Thromboseprophylaxe ist besonders bei immobilisierten Patienten sinnvoll. Ein „Magenschutz“ sollte immer gegeben werden. Sollte in Ausnahmefällen eine intravenöse Gabe nicht möglich sein, ist nach neueren Erkenntnissen eine orale Hochdosistherapie mit 500 mg Methylprednisolon über fünf Tage und anschließender oraler Ausschleichphase möglich (15).

Bei unzureichendem Ansprechen der Schubsymptome auf die intravenöse Steroidgabe sollte nach zwei Wochen eine erneute Gabe von Methylprednisolon, eventuell mit $2 \mathrm{~g}$ täglich intravenös erfolgen. Dies muss unter o.g. Prophylaxe und regelmäßigen Kontrolluntersuchungen (Blutdruck- und Blutzucker) durchgeführt werden. Hat diese Therapie keinen ausreichenden Erfolg, ist bei einer deutlichen Beeinträchtigung des Patienten eine Plasmaaustauschtherapie (19) zu diskutieren (Abb. 1). Es bietet sich an, hierzu mit einem erfahrenen MSZentrum Kontakt aufzunehmen.

\section{Basistherapeutika}

Zahlreiche Studien haben die Wirksamkeit von verschiedenen Substanzen zur Schubprophylaxe und zur Verzögerung der Krankheitsprogression gezeigt. Das erste in Deutschland zugelassene Präparat war 1995 Interferon- $\beta$ 1b (Betaferon ${ }^{\circledR}$ ) zur Behandlung der schubförmig-remittierenden Verlaufsform. Die Anwendung erfolgt durch subkutane Gabe jeden zweiten Tag. Dasselbe Präparat erhielt 1998 eine erweiterte Zulassung zur Therapie auch bei einer sekundär progredient verlaufenden MS. Seit 1997 stehen für die Behandlung der schubförmigen MS auf dem deutschen Markt außerdem das einmal wöchentlich intramuskulär zu applizierende Interferon- $\beta$ 1a (Avonex $^{\circledR}$ ) und seit 1998 das dreimal wöchentlich subkutan zu applizierende Interferon- $\beta$ 1a (Rebif ${ }^{\circledR}$ ) zur Verfügung. Das auf anderen Wirkmechanismen beruhende Präparat Glatirameracetet (Copaxone ${ }^{\circledR}$ ) wurde 2001 in Deutschland für die Behandlung der schubförmigen MS zugelassen und muss täglich subkutan injiziert werden.

\section{Interferon- $\beta$}

Die immunmodulatorische Wirkung der Interferone beruht unter anderem auf der Unterdrückung proinflammatorischer Zytokine wie Interferon- $\beta$ und Tumor-NekroseFaktor- $\beta$, der Induktion immunmodulatorischer TH2-Zytokine wie Interleukin-10, der Steigerung der TLymphozyten-Suppressor-Aktivität, der Induktion löslicher Adhäsionsmoleküle sowie einer Herabsetzung der Permeabilität der Blut-Hirnschranke (6).

Bei der MS handelt es sich nicht nur um eine entzündliche und

\section{Abb.1 Sekundärprophylaktische Intervalltherapie des schweren MS-Schubes (schwere Optikusneuritis, Hirnstamm- oder spinale Symptomatik)}

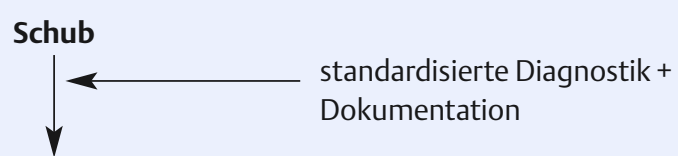

1000 mg/d über 3-5 Tage

(orales Ausschleichen)

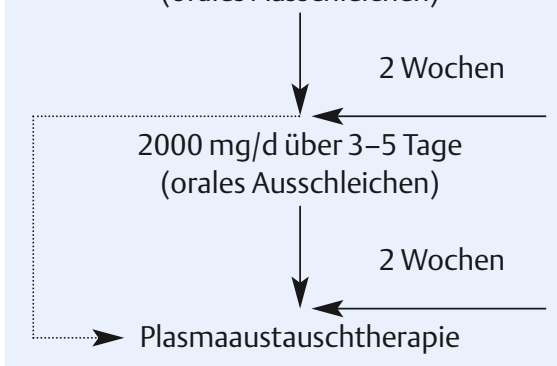

standardisierte Diagnostik + Dokumentation (MS-Zentrum) (Oliver et al. Neurology, 1998)

standardisierte Diagnostik + Dokumentation (MS-Zentrum) (Weinshenker et al. Ann Neurol, 1999) 
demyelinisierende Erkrankung. Eine wichtige Rolle scheinen auch neurodegenerative Prozesse zu spielen (9). Neue Studien haben deutlich gemacht, dass die Anzahl degenerierter Axone in den inflammatorischen Läsionen am höchsten ist. Anhand von Biopsien konnte außerdem gezeigt werden, dass vor allem im ersten Erkrankungsstadium bereits eine massive Degeneration von Axonen stattfindet (8). Die sich daraus ableitende Schlussfolgerung eines frühen Behandlungsbeginns konnte mit der ETOMS- (5) und der CHAMPS-Studie (7) untermauert werden: bei Therapiebeginn nach dem ersten klinischen Ereignis und dem Nachweis klinisch stummer Läsionen im zerebralen MRT sowie Ausschluss anderer möglicher Ursachen konnte der zweite Schub signifikant hinausgezögert werden. Da-

\section{Abb. 2 Immunmodulatorische Stufen- therapie der MS (MSTKG 2000) (12)}

Cyclophasphamid
Mitoxantron
Interferon Beta, GLAT
Azathioprin, IVIG
Methylprednisolon
GLAT = Clatirameracetat;
IVIG = intravenöse Immunglobuline

\section{Tab. 1 MSTKG-Empfehlungen zur Immunprophylaxe nach einem Erstsymptom}

Intrathekale lgG-Synthese

Ausschluss anderer Ursachen

Mindestens eines der folgenden Kriterien:

- Funktionell deutlich beeinträchtigende Schubsymptomatik, die sich unter Kortisonstoßtherapie innerhalb von 6 Monaten nicht ausreichend zurückbildet oder

- Gadolinium anreichernde Herde innerhalb von 6 Monaten oder

- neue Herde im T2-gewichteten MRT innerhalb von 6 Monaten oder

$\bullet \geq 6$ Herde im MRT raus leiteten sich die in Tabelle 1 aufgeführten MSTKG-Empfehlungen zur Immunprophylaxe nach einem Erstsymptom ab.

Die zu erwartenden Nebenwirkungen sind bei allen drei Interferon-Präparaten ähnlich. So kommt es bei mehr als der Hälfte der Patienten insbesondere in der Einstellungsphase, die einschleichend sein sollte, zu so genannten „flu-like symptoms“, zu grippeähnlichen Nebenwirkungen wie Fieber, Myalgien, Kopfschmerzen und Abgeschlagenheit. Dem kann mit der Gabe von nichtsteroidalen Antirheumatika (z.B. Paracetamol oder Ibuprofen) und durch abendliche Injektionen begegnet werden. Andere typische Nebenwirkungen sind eine Lymphopenie, ein Transaminasenanstieg sowie, besonders bei der subkutanen Gabe, Hautreaktionen an der Injektionsstelle. In seltenen Fällen kann es zu einer Verstärkung von Spastik und Fatigue kommen. Das Auftreten von depressiven Störungen im Rahmen einer Interferon- $\beta$-Therapie wird kontrovers diskutiert (17).

Unter der Behandlung mit Interferon- $\beta$ kann es zum Auftreten von neutralisierenden Antikörpern kommen, deren praktische Bedeutung bisher jedoch noch unklar ist. Im Einzelfall erleichtert jedoch der reproduzierbare Nachweis dieser Antikörper beim klinischen Eindruck eines Therapieversagens die Entscheidung zum Wechsel der Therapiestrategie. Bisher gibt es keine allgemein gültige Definition eines Therapieversagens bei der Multiplen Sklerose.

Aus den vorliegenden Studiendaten zum schubförmigen Verlauf ergeben sich aber Hinweise, dass eine nach drei bis sechs Monaten bestätigte Krankheitsprogression um einen Punkt auf der EDSS-Skala oder um 0,5 Punkte bei Ausgang-EDSSWerten von sechs bzw. sieben hierfür einen verwertbaren Parameter darstellt. Im Einzelfall ist aber auch ein Vergleich mit der Progressionsdynamik/Schubrate vor Therapie angebracht (11).

Eine Änderung der Therapiestrategie kann prinzipiell eine Dosissteigerung, ein Wechsel der immuntherapeutischen Substanz oder eine Kombinationstherapie sein.

\section{Glatirameracetat}

Dieses aus vier Aminosäuren bestehende Peptidgemisch zeigt eine Kreuzreaktivität zu basischem Myelinprotein MBP auf T- und B-Zellebene und bindet an den MHC IIKomplex antigenpräsentierender Zellen. Dadurch kommt es zu einer Blockade von Bindungstellen für MBP an MHC-II-Molekülen. Darüber hinaus bewirkt es die Induktion von protektiven T-Zellen (TH2-Typ), die Verschiebung des Zytokinmusters zu „Vorteilhaften“ immunmodulierenden Zytokinen wie IL-4, IL-6 und IL10 und die Forderung der Neurotrophinsekretion als möglichem neuroprotektiven Wirkprinzip (6).

Die Rate der Therapieerfolge ist mit denen der Interferon- $\beta$-Therapie vergleichbar. Nebenwirkungen treten jedoch seltener auf und betreffen vorrangig lokale Nebenwirkungen an der Injektionsstelle, wie Rötungen, Schwellungen, Schmerzen und Gewebsindurationen, die jedoch im Verlauf der Therapie in der Regel abnehmen. In den ersten sechs Monaten nach Therapiebeginn kann es bei zirka $15 \%$ der Patienten zur so genannten „systemischen Postinjektionsreaktion (SPIR)“ kommen, die wahrscheinlich auf einer Histaminfreisetzung beruht und mit Herzrasen, Atemnot, thorakalem Druckgefühl, Schweißausbrüchen und Blutdruckabfall einhergeht. In der Regel klingen die Symptome nach 30 Sekunden bis 30 Minuten ohne Folgen wieder ab. Studiendaten zeigen bisher den besten therapeutischen Effekt bei Beginn der Therapie im niedrigen EDSS-Bereich (0-2), sodass der bevorzugte therapeutische Einsatz im frühen schubförmigen Stadium liegt.

\section{Alternative Basistherapien Azathioprin}

Azathioprin, ein Purinanalog, wirkt als ein Immunsuppressor und kann oral eingenommen werden. Es greift in den Purinnukleotid-Stoffwechsel ein und wirkt über einen Metabolit durch Hemmung der Aktivierung und Teilung von Lymphozyten immunsuppressiv (1). Zugelassen zur Therapie der schubförmigen MS ist es in Deutschland seit 2001. Es wurde jedoch bereits seit 
Ende der 60er-Jahre bei der Behandlung der MS eingesetzt. Auch wenn die überwiegend in den 70er und 80er-Jahren publizierten offenen Therapiestudien nicht mehr den heutigen Anforderungen an Therapiestudien genügen, so konnte in einer Metaanalyse eine Wirksamkeit von Azathioprin bei MS sowohl auf die Anzahl der Schübe als auch auf die Krankheitsprogression bei schubförmiger MS gezeigt werden (6).

Leider liegen für Azathioprin bis heute keine doppelblinden plazebokontrollierten Studien mit Berücksichtigung von MRT-Daten vor. Dies ist jedoch nicht der einzige Grund für das eingeschränkte Indikationsspektrum dieses Medikaments. So wird bei einer langjährigen Azathioprinbehandlung ein erhöhtes Malignomrisiko (v.a. aus dem hämatologischen Formenkreis) diskutiert. Wegen der Gefahr von schweren bakteriellen Infektionen, insbesondere einer Endokarditis lenta oder einer Tuberkulose sind Röntgenkontrollen des Thorax im Verlauf empfehlenswert. Außerdem sind regelmäßige Kontrollen von Differenzialblutbild (toxische hämatologische Veränderungen) und Leberwerten notwendig. In seltenen Fällen kommt es nach Einnahme von Azathioprin zu unstillbarem Erbrechen, das therapeutisch nicht zu beeinflussen ist und zu einem Abbrechen der Therapie zwingt. Da eine Teratogenität nicht sicher auszuschließen ist, muss unter der Therapie und bis sechs Monate nach Absetzen eine sichere Kontrazeption - wie auch unter einer Inteferon- $\beta$-Therapie - gewährleistet sein. Indiziert erscheint es deshalb nur dann, wenn ein Patient unter keinen Umständen bereit ist, ständige Injektionen (Spritzenphobie) in Kauf zu nehmen. Die Dosis richtet sich nach der absoluten Lymphozytenzahl im Serum, wobei ein Wert zwischen 800-1200/Ml angestrebt wird. Die Standarddosis von $2,5 \mathrm{mg} / \mathrm{kg}$ Körpergewicht sollte eingeschlichen werden.

\section{Intravenöse Immunglobuline}

Intravenöse, polyvalente 7S- Immunglobuline (IVIG) verfügen über eine Vielzahl von immunmodulato- rischen Eigenschaften. So kommt es zur Neutralisierung von Komplement-vermittelten Effekten und Hemmung der überschießenden Komplementaktivierung, einer Blockade von Fc-Rezeptoren, einer verminderten Produktion proinflammatorischer Zytokine sowie zur direkten Neutralisierung inhibitorischer Antikörper von B-Lymphozyten. Der genaue Wirkmechanismus bei der Multiplen Sklerose ist bisher nicht bekannt. Vier klinische Studien der Klasse II- und III-Evidenz sprechen bisher für eine Wirksamkeit von IVIG bei der schubförmigen MS. Die Ende 2001 erstmals und bisher nur auf Kongressen vorgestellte ESIMS-Studie (Klasse-I-Evidenz) zur Behandlung der sekundär progredienten MS war jedoch in allen Endpunkten negativ ausgegangen. Weder zeigte sich ein Effekt auf die Krankheitsprogression noch auf die Schubrate in der Phase der sekundären Progression (16). Dies hat sowohl zu einer Verunsicherung beim Einsatz dieses Präparates als auch zu Konflikten mit den Kostenträgern geführt. Die Ergebnisse drei weiterer multizentrischer, kontrollierter, doppelblinder Therapiestudien stehen derzeit noch aus. Bis dahin wurden die Empfehlungen der MSTKG hinsichtlich des Einsatzes von IVIG bei MS nicht geändert (13). Die Behandlung ist relativ nebenwirkungsarm. Zu nennen sind unter anderem Hautexantheme, kardiovaskuläre Komplikationen, Kopfschmerzen und sehr selten anaphylaktische Reaktionen oder Thrombo- sen. Die Nebenwirkungen können durch eine langsame Infusionsgeschwindigkeit von unter $10 \mathrm{~g} \mathrm{Im-}$ munglobulinen/Stunde reduziert werden (20). Aufgrund der geringen Nebenwirkungsrate und der fehlenden Zulassung anderer Präparate ist die Behandlung mit IVIG besonders bei Kindern und Jugendlichen unter 18 Jahren und bei einer notwendigen Behandlung während und nach der Schwangerschaft indiziert (offlabel-use). Derzeit ist aus unserer Sicht eine initiale Dosis von jeweils $0,4 \mathrm{~g} / \mathrm{kg}$ Körpergewicht an fünf aufeinander folgenden Tagen gefolgt von monatlichen Booster-Infusionen (einmal 0,4 g/kg Körpergewicht) zu empfehlen.

\section{Eskalationstherapie}

Bei Therapieversagen bzw. einem Nachlassen der Wirksamkeit der vorgenannten Basistherapien mit unveränderter oder zunehmender Schubfrequenz bzw. Übergang in eine chronisch progrediente Verlaufsform mit stetiger Krankheitsprogression muss die Indikation zur Eskalationstherapie (Mitoxantron, Cyclophosphamid) überprüft werden.

\section{Mitoxantron}

Mitoxantron ist ein synthetisches Anthracenedoin, das in der Onkologie überwiegend bei der Therapie des Mammakarzinoms und verschiedener Lymphome eingesetzt wird. Es interkaliert in die DNA, führt hier zu Einzel- und Doppelstrangbrüchen und so zu einer Hem-

\section{Tab. 2 Immuntherapeutische Möglichkeiten bei MS}

\begin{tabular}{ll}
$\begin{array}{l}\text { Schubtherapie } \\
\text { Sekundärprophylaxe } \\
\text { (Basistherapie) }\end{array}$ & $\begin{array}{l}\text { Hochdosiertes Methylprednisolon } \\
\text { Plasmapherese bei Steroid-Nonresponse }\end{array}$ \\
$\begin{array}{l}\text { Therapie 1. Wahl: Interferon Beta* } \\
\text { Glatirameracetat* } \\
\text { Therapie 2. Wahl: Azathioprin* (max. 10 Jahre) } \\
\text { Immunglobuline }\end{array}$ \\
\hline $\begin{array}{l}\text { Sekundärprophylaxe } \\
\text { (Eskalationstherapie) }\end{array}$ & $\begin{array}{l}\text { Mitoxantron* (max. } 140 \mathrm{mg} / \mathrm{m} 2) \\
\text { Cyclophosphamid }\end{array}$ \\
\hline $\begin{array}{l}\text { Therapie maligner } \\
\text { Verlaufsformen }\end{array}$ & $\begin{array}{l}\text { Mitoxantron* (max. } 140 \mathrm{mg} / \mathrm{m} 2) \\
\text { Cyclophosphamid } \\
\text { Plasmapherese }\end{array}$ \\
\hline $\begin{array}{l}\text { Therapie bei primär } \\
\text { progredienter MS }\end{array}$ & Methylprednisolon-Intervalltherapie \\
* zugelassene Therapieverfahren
\end{tabular}


mung der Nukleinsäuresynthese. Auf diese Weise kommt es zu einer Suppression von proliferierenden Zellen, unter anderem von B-Lymphozyten (6). Seit Ende 2002 ist Mitoxantron (Ralenova ${ }^{\circledR}$ ) auch für die Behandlung der schubförmigen und sekundär progredienten MS in Deutschland zugelassen. Wegen der potenziell kardiotoxischen Wirkung ist der Einsatz dieses Präparates jedoch begrenzt und darf nur unter halbjährlichen EKG- und Echokardiographiekontrollen bis zu einer kumulativen Gesamtdosis von maximal $140 \mathrm{mg} / \mathrm{m}^{2}$ Körperoberfläche verabreicht werden, was in Abhängigkeit von der initialen Dosis und bei eventuellem Reduzieren der Dosierung nach fünf Einzeldosen bei stabilem Verlauf einem ungefähren Behandlungszeitraum von fünf bis sechs Jahren entspricht.

Neben den seltenen, jedoch irreversiblen Kardiomypopathien kann es unter Mitoxantron zu Übelkeit mit Erbrechen, Haarausfall, sekundärer Amenorrhoe oder zu einer Knochenmarkssuppression kommen. Die Therapie sollte in dreimonatigem Abstand nach Ausschluss kardialer Vorschädigung mit einer initialen Dosis von $10-12 \mathrm{mg} / \mathrm{m}^{2}$ Körperoberfläche begonnen werden. Bei klinischer Stabilität kann nach fünf Zyklen nach eigenen Erfahrungen eine Halbierung der Dosis erwogen werden $\left(5 \mathrm{mg} / \mathrm{m}^{2}\right.$ Körperoberfläche alle drei Monate). Vor und nach Mitoxantron-Applikation sollte ein Antiemetikum (z.B. Ondansetron) verabreicht werden (11).

\section{Cyclophosphamid}

Dieses Alkylans lagert kovalent Alkylgruppen an die DNA an und wirkt somit während des gesamten Zellzyklus zytotoxisch. Die konsekutive immunsuppressive Wirkung führt zu einer Reduktion von B-Lymphozyten sowie von CD4- und CD8T-Lymphozyten. Wichtige Nebenwirkungen dieser Therapie sind gastrointestinale Störungen mit Übelkeit und Erbrechen. Dem sollte mit einer antiemetischen Prophylaxe vor und nach den Infusionen begegnet werden. Besonders gefürchtet ist eine hämorrhagische Zystitis, die eine Gabe von 2-Mercapto-Ethan- sulfonat sowie 3-4 Liter Flüssigkeitsaufnahme am Infusionstag erforderlich machen. Des Weiteren kommt es zu Blutbildveränderungen, weshalb nach dem jeweiligen Behandlungszyklus (Tag 7 und 14) engmaschige Blutbildkontrollen notwendig sind. Gelegentlich kommt es unter der Therapie zu einer reversiblen Alopecia (15). Zur Behandlung der MS ist eine initiale Dosis von 600 $\mathrm{mg} / \mathrm{m}^{2}$ Körperoberfläche als Kurzinfusion alle vier bis sechs Wochen zu empfehlen. Zur individuellen Dosisanpassung ist die Bestimmung des Lymphozytennadirs nach der Therapie sinnvoll.

Der Einsatz von Cyclophosphamid in der Behandlung der MS ist dann indiziert, wenn eine Unverträglichkeit oder Kontraindikationen gegen Mitoxantron vorliegen. Eine Zulassung des Präparates zur Therapie der MS liegt bisher nicht vor:

\section{Zyklische Glukokotikosteroid- Applikation}

Im Gegensatz zur schubförmigen und zur sekundär progredienten Verlaufsform der MS fehlen beim primär progredienten Verlauf klare Therapiekonzepte auf dem Boden von Studien. Als Therapieoptionen kommt eine probatorische zyklische Steroidpulstherapie mit $1 \mathrm{~g}$ Methylprednisolon täglich an fünf aufeinander folgenden Tagen alle drei Monate infrage (4).

\section{Zukunftsrelevante Therapie- ansätze}

Neue Erkenntnisse zur Immunpathologie, Fortschritte in der Biotechnologie und verbesserte bildgebende Verfahren führen zu immer neuen Therapieansätzen bei der MS. Erfolgsversprechende, sich derzeit in der klinischen Prüfung befindliche Substanzen sind VLA-4-Antikörper, Phosphodiesterase-IV-Inhibitoren, Statine sowie CCR1- Antagonisten. Für alle diese Substanzen ist eine antiinflammatorische Wirkung im Tiermodell nachgewiesen, sodass aktuell Phase-II-Studien zur Behandlung bei MS durchgeführt werden (2).

Allerdings muss an dieser Stelle auf die zahlreichen negativen Studi- enerfahrungen mit erfolgsversprechenden Substanzen hingewiesen werden. Trotz rationaler Therapiekonzepte, überzeugender tierexperimenteller Voruntersuchungen oder positiven Erfahrungen bei anderen Autoimmunerkrankungen gelang für viele Substanzen kein Wirksamkeitsnachweis bei MS oder die Untersuchungen mussten wegen unvorhergesehener Nebenwirkungen vorzeitig abgebrochen werden. Beispiele hierfür sind die Studien mit der immunmodulatorischen Substanz Linomid, deren Erprobung im Phase-II-Stadium wegen schwerwiegender kardiovaskulärer Nebenwirkungen abgebrochen werden musste. Ein anderes Beispiel betrifft das aus der Rheumatologie bekannte immunmodulatorische und antiinflammatorisch wirksame Sulfasalazin, das nach anfänglich guter Wirksamkeit bei MS in einem Beobachtungszeitraum von drei Jahren schließlich zu keiner signifikanten Verzögerung der Krankheitsprogression gegenüber Plazebo führte (18).

Klinisch erfolgreiche Therapieprinzipien neuroprotektiver Art gegen den neurodegenerativen Teil des Pathomechanismus der MS stehen noch aus.

There is almost no other neurological disease, compared with multiple sclerosis, where such substantial advances in therapy have been made over the last 5 years. Beta-interferons and glatiramer acetate are basic therapeutic agents for different courses of multiple sclerosis. Even in cases with treatment failures or with dramatic courses of disease there are expanding evidence-based capabilities for the treatment escalation. Such treatments consist of immunsuppressive agents like mitoxantrone or cyclophosphamide, but also plasmapheresis can be useful in some cases. Newly developed treatment strategies not only try to suppress or to modulate the immune system; neuroprotection and regeneration are treatment targets of future therapies.

\section{Key Words:}

multiple sclerosis - therapy - betainterferons - glatiramer acetate immunsuppressive agents 


\section{Literatur}

1. Adler S, Zettl UK. Kausalorientierte Therapieoptionen be Multipler Sklerose. Nervenheilkunde 2002; 2: 74-80

2. Aktas O, Wandinger K-P, Zipp F. Therapeutisch relevante MSForschung - ein Ausblick. Nervenheilkunde 2003; 4: 46-50

3. Beck RW, Cleary PA, Anderson MM et al. A randomised, controlled trial of corticosteroids in the treatment of acute optic neuritis. The Optic Neuritis Study Group. N Engl J Med 1992; 326: $581-588$

4. Cazzato G, Mesiano T, Antonello R et al. Double-blind, placebo-controlled, randomised, crossover trial of high dose methylprednisolone in patients with chronic progressive form of multiple sclerosis. Eur Neurol 1995; 35: 193-198

5. Comi G, Filipi M, Barkhof $F$ et al. Effect of early interferon treatment on conversion to definite multiple sclerosis: a randomised study. Lancet 2001; 357: 1576-1582

6. Gold R, Hartung H.-P. Aktueller Therapieüberblick und zukünftige Therapiestrategien der Multiplen Sklerose. Nervenheilkunde 2003; 4: 16-24

7. Jacobs LD, Beck RW, Simon JH et al. Intramuscular interferon beta-1a therapy initiated during a first demyelinating event in multiple sclerosis. CHAMPS Study Group. N Engl J Med 2000; 343: 898-904

8. Kuhlmann T, Lingfeld G, Bitsch A et al. Acute axonal damage in multiple sclerosis is most extensive in early disease stages and decreases over time. Brain 2002;125: 2202-2212

9. Lassmann H. Mechanisms of demyelination a tissue destruction in multiple sclerosis. Clin Neurol Neurosurg 2002; 104 168-171

10. McDonald WI, Compston A, Edan G et al. Recommended diagnostic criteria for multiple sclerosis: guidelines from the International Panel on the diagnosis of multiple sclerosis. Ann Neurol 2001, 50: 121-127

11. MSTKG. Immunmodulatorische Stufentherapie der multiplen Sklerose. Nervenarzt 1999; 70: 371-386

12. MSTKG. Immunmodulatorische Stufentherapie der multiplen Sklerose. Nervenarzt 2001; 72: 150-157

13. MSTKG. Immunmodulatorische Stufentherapie der Multiplen Sklerose. Nervenarzt 2002; 73: 556-563

14. Neuhaus O, Archelos J], Hartung HP. Immunmodulation in multipler sclerosis: from immunsupression to neuroproduction, Trends Pharmacol Sci 2003; 24: 131-138

15. Sellebjerg F, Frederiksen JL, Nielsen PM, Olesen J. Doubleblind, randomised, placebo-controlled study of oral, high-dose methylprednisolone in attacks of MS. Neurology 1998 Aug; 51: 529-34

16. Soelberg-Soerensen P. ESIMS. European Chacot Foundation; Venice 21.10.2001

17. Walter EU, Hohlfeld R. Multiple Sclerosis, Side effects of interferon beta therapy and their management, Neurology 1999; 53: 1622-1627

18. Wiendl H, Hohlfeld R, Therapieansätze bei der Multiplen Sklerose-Lektionen aus fehlgeschlagenen und abgebrochenen Studien, Nervenheilkunde 2003; 4: 25-31

19. Weinshenker BG, O'Brien PC, Petterson TM et al. A randomised trial of plasma exchange in acute central nervous system inflammatory demyelinating disease, Ann Neurol 1999; 46: 878-886

20. Wittstock M, Benecke R, Zettl U.K. Therapy with intravenous immunglobulins: Complications and side effects. Eur Neurol 2003 (in press)

21. Zettl UK, Mix E (Hrsg.), Multiple Sklerose, Springer Verlag, 2001

\section{Korrespondenzadresse:}

Dr. Isabel Ringel

Klinik und Poliklinik für Neurologie

Zentrum für Nervenheilkunde

Gehlheimer Str. 20

18147 Rostock

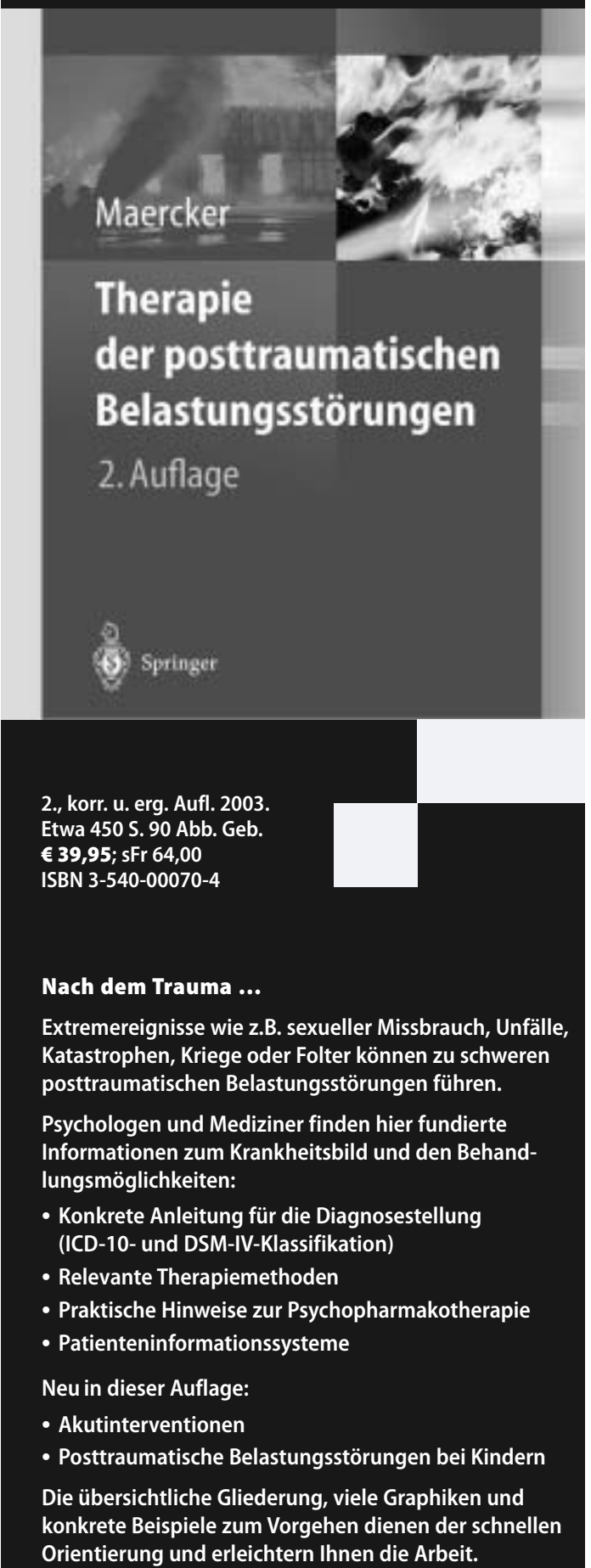

Orientierung und erleichtern Ihnen die Arbeit.

.. die Patienten adäquat versorgen!

\section{Springer Fachbücher. * \\ Die spannenden Seiten der Medizin.}

Bestellen Sie jetzt bei Ihrer Buchhandlung!

Weitere Infos unter www.springer.de/medizin 